\title{
ON NEW GENERALIZATIONS OF HILBERT'S INEQUALITIES
}

\author{
ZHONGXUE LÜ
}

\begin{abstract}
In this paper, some generalizations of Hilbert's inequalities are shown by introducing
\end{abstract} two real functions $\phi(x)$ and $\psi(x)$.

\section{Introduction}

The following inequalities are well-known as Hilbert's integral inequality:

$$
\begin{gathered}
\int_{0}^{\infty} \int_{0}^{\infty} \frac{f(x) g(y)}{x+y} d x d y \leq \pi\left(\int_{0}^{\infty} f^{2}(x) d x \int_{0}^{\infty} g^{2}(y) d y\right)^{1 / 2} \\
\int_{0}^{\infty}\left(\int_{0}^{\infty} \frac{f(x)}{x+y} d x\right)^{2} d y \leq \pi^{2} \int_{0}^{\infty} f^{2}(x) d x
\end{gathered}
$$

where $\pi$ is the best value (Cf. [1, Chap 9]). Their associated double series forms are as follows, respectively. If $\left\{a_{m}\right\}$ and $\left\{b_{n}\right\}$ are sequences of real numbers such that $0<$ $\sum_{m=1}^{\infty} a_{m}^{2}<\infty$ and $0<\sum_{n=1}^{\infty} b_{n}^{2}<\infty$, then

$$
\begin{gathered}
\sum_{m=1}^{\infty} \sum_{n=1}^{\infty} \frac{a_{m} b_{n}}{m+n} \leq \pi\left(\sum_{m=1}^{\infty} a_{m}^{2} \sum_{n=1}^{\infty} b_{n}^{2}\right)^{1 / 2} \\
\sum_{n=1}^{\infty}\left(\sum_{m=1}^{\infty} \frac{a_{m}}{m+n}\right)^{2} \leq \pi^{2} \sum_{m=1}^{\infty} a_{m}^{2}
\end{gathered}
$$

In recent years, $\mathrm{Hu}$ [2], Gao [3] and Kuang [4] gave some distinct improvements of (1) and (3), and Gao [5] gave (3) a strengthened version. Yang Bicheng [6] gave interesting generalization of (1) by introducing parameters $\lambda \in(0,1]$.

$$
\int_{0}^{\infty} \int_{0}^{\infty} \frac{f(x) g(y)}{(x+y)^{\lambda}} d x d y<B\left(\frac{\lambda}{2}, \frac{\lambda}{2}\right)\left(\int_{0}^{\infty} t^{1-\lambda} f^{2}(t) d t \int_{0}^{\infty} t^{1-\lambda} g^{2}(t) d t\right)^{1 / 2}
$$

where $B(p, q)$ is the $\beta$ function.

Received October 23, 2002; revised December 30, 2002.

2000 Mathematics Subject Classification. 26D15.

Key words and phrases. Hilbert's inequality, Hölder's inequality. 
Yang Bicheng [7] made some generalizations of (1) and (3) and (5) by introducing three parameters, $A, B$ and $\lambda$.

$$
\begin{gathered}
\int_{0}^{\infty} \int_{0}^{\infty} \frac{f(x) g(y)}{(A x+B y)^{\lambda}} d x d y<\frac{1}{(A B)^{\lambda / 2}} B\left(\frac{\lambda}{2}, \frac{\lambda}{2}\right) \\
\times\left(\int_{0}^{\infty} x^{1-\lambda} f^{2}(x) d x \int_{0}^{\infty} y^{1-\lambda} g^{2}(t) d y\right)^{1 / 2} \\
\int_{0}^{\infty} y^{\lambda-1}\left(\int_{0}^{\infty} \frac{f(x)}{(A x+B y)^{\lambda}} d x\right)^{2} d y<\frac{1}{(A B)^{\lambda}}\left(B\left(\frac{\lambda}{2}, \frac{\lambda}{2}\right)\right)^{2} \int_{0}^{\infty} x^{1-\lambda} f^{2}(x) d x \\
\sum_{m=1}^{\infty} \sum_{n=1}^{\infty} \frac{a_{m} b_{n}}{(A m+B n)^{\lambda}}<\frac{1}{(A B)^{\lambda / 2}} B\left(\frac{\lambda}{2}, \frac{\lambda}{2}\right)\left(\sum_{m=1}^{\infty} m^{1-\lambda} a_{m}^{2} \sum_{n=1}^{\infty} n^{1-\lambda} b_{n}^{2}\right)^{1 / 2} \\
\sum_{n=1}^{\infty} n^{\lambda-1}\left(\sum_{m=1}^{\infty} \frac{a_{m}}{(A m+B n)^{\lambda}}\right)^{2}<\frac{1}{(A B)^{\lambda}}\left(B\left(\frac{\lambda}{2}, \frac{\lambda}{2}\right)\right)^{2} \sum_{m=1}^{\infty} m^{1-\lambda} a_{m}^{2}
\end{gathered}
$$

Where $A, B, \lambda>0$.

In this paper, we show some new generalizations on above inequalities by introducing two real functions $\phi(x)$ and $\psi(x)$.

First we introduce some Lemmas:

Lemma 1.([9]) Let $a<1, \lambda>0$, define $h(y)$ as

$$
h(y)=y^{-1+a} \int_{0}^{y} \frac{1}{(1+u)^{\lambda}}\left(\frac{1}{u}\right)^{a} d u, \quad y \in(0,1]
$$

Then $h(y)>h(1)(0<y<1)$.

Use the same way, we get the following Lemma.

Lemma 2. Let $a<1, \lambda>0$, define $h(y)$ as

$$
h(y)=y^{-1+a} \int_{0}^{y} \frac{1}{1+u^{\lambda}}\left(\frac{1}{u}\right)^{a} d u, \quad y \in(0,1] .
$$

Then $h(y)>h(1)(0<y<1)$.

Lemma 3. Let $p>1,1 / p+1 / q=1, \phi(x)$ and $\psi(x)$ are differentiable functions, and $\phi(0) \geq 0, \phi^{\prime}(x)>0, \psi(0) \geq 0, \psi^{\prime}(x)>0, \phi^{\prime}(x)$ and $\psi^{\prime}(x)$ has infimum, respectively, $\lambda>2-\min \{p, q\}$, define $\omega_{1}(\phi, \psi, q, \lambda, x)$ as

$$
\omega_{1}(\phi, \psi, q, \lambda, x)=\int_{0}^{\infty} \frac{1}{(\phi(x)+\psi(y))^{\lambda}}\left(\frac{\phi(x)}{\psi(y)}\right)^{\frac{2-\lambda}{q}} d y, \quad x>0
$$

then

$$
\omega_{1}(\phi, \psi, q, \lambda, x) \leq \frac{\phi^{1-\lambda}(x)}{\inf \left\{\psi^{\prime}(y)\right\}} B\left(\frac{q+\lambda-2}{q}, \frac{p+\lambda-2}{p}\right) .
$$


Proof. Putting $u=\frac{\psi(y)}{\phi(x)}$, we have

$$
\begin{aligned}
\omega_{1}(\phi, \psi, q, \lambda, x) & \leq \frac{\phi^{1-\lambda}(x)}{\inf \left\{\psi^{\prime}(y)\right\}} \int_{0}^{\infty} \frac{1}{(1+u)^{\lambda}}\left(\frac{1}{u}\right)^{\frac{2-\lambda}{q}} d u \\
& =\frac{\phi^{1-\lambda}(x)}{\inf \left\{\psi^{\prime}(y)\right\}} B\left(\frac{q+\lambda-2}{q}, \frac{p+\lambda-2}{p}\right) .
\end{aligned}
$$

The lemma is proved.

Lemma 4. Let $p>1,1 / p+1 / q=1, \phi(x)$ and $\psi(x)$ are differentiable functions, and $\phi(0) \geq 0, \phi^{\prime}(x)>0, \psi(0) \geq 0, \psi^{\prime}(x)>0, \phi^{\prime}(x)$ and $\psi^{\prime}(x)$ has infimum, respectively, $\lambda>2-\min \{p, q\}$, define $\omega_{2}(\phi, \psi, q, \lambda, x)$ as

$$
\omega_{2}(\phi, \psi, q, \lambda, x)=\int_{0}^{\infty} \frac{1}{(\phi(x))^{\lambda}+(\psi(y))^{\lambda}}\left(\frac{\phi(x)}{\psi(y)}\right)^{\frac{2-\lambda}{q}} d y, \quad x>0
$$

then

$$
\omega_{2}(\phi, \psi, q, \lambda, x) \leq \frac{\phi^{1-\lambda}(x)}{\lambda \inf \left\{\psi^{\prime}(y)\right\}} B\left(\frac{q+\lambda-2}{q \lambda}, 1-\frac{q+\lambda-2}{q \lambda}\right)
$$

Proof. Putting $u=\frac{\psi(y)}{\phi(x)}$, we have

$$
\begin{aligned}
\omega_{2}(\phi, \psi, q, \lambda, x) & \leq \frac{\phi^{1-\lambda}(x)}{\inf \left\{\psi^{\prime}(y)\right\}} \int_{0}^{\infty} \frac{1}{1+u^{\lambda}}\left(\frac{1}{u}\right)^{\frac{2-\lambda}{q}} d u \\
& =\frac{\phi^{1-\lambda}(x)}{\lambda \inf \left\{\psi^{\prime}(y)\right\}} B\left(\frac{q+\lambda-2}{q \lambda}, 1-\frac{q+\lambda-2}{q \lambda}\right) .
\end{aligned}
$$

The lemma is proved.

\section{Main Results}

Now we introduce main results.

Theorem 1. Let $p>1,1 / p+1 / q=1, f(x), g(x) \geq 0, \phi(x)$ and $\psi(x)$ are differentiable functions, and $\phi(0) \geq 0, \phi^{\prime}(x)>0, \psi(0) \geq 0, \psi^{\prime}(x)>0, \phi^{\prime}(x)$ and $\psi^{\prime}(x)$ has infimum, respectively, $\lambda>2-\min \{p, q\}$, such that $0<\int_{0}^{\infty} \phi^{1-\lambda}(t) f^{p}(t) d t<\infty$ and $0<\int_{0}^{\infty} \phi^{1-\lambda}(t) g^{q} d t<\infty$. Then

$$
\begin{aligned}
\int_{0}^{\infty} \int_{0}^{\infty} \frac{f(x) g(y)}{(\phi(x)+\psi(y))^{\lambda}} d x d y \leq & \frac{B\left(\frac{q+\lambda-2}{q}, \frac{p+\lambda-2}{p}\right)}{\left(\inf \left\{\phi^{\prime}(x)\right\}\right)^{\frac{1}{q}}\left(\inf \left\{\psi^{\prime}(y)\right\}\right)^{\frac{1}{p}}} \\
& \times\left(\int_{0}^{\infty} \phi^{1-\lambda}(x) f^{p}(x) d x\right)^{\frac{1}{p}}\left(\int_{0}^{\infty} \psi^{1-\lambda}(y) g^{q}(y) d y\right)^{\frac{1}{q}}
\end{aligned}
$$




$$
\begin{aligned}
\int_{0}^{\infty} \psi^{(\lambda-1)(p-1)}(y)\left(\int_{0}^{\infty} \frac{f(x)}{(\phi(x)+\psi(y))^{\lambda}} d x\right)^{p} d y \leq & \frac{\left(B\left(\frac{q+\lambda-2}{q}, \frac{p+\lambda-2}{p}\right)\right)^{p}}{\left(\inf \left\{\phi^{\prime}(x)\right\}\right)^{p-1} \inf \left\{\psi^{\prime}(y)\right\}} \\
& \times \int_{0}^{\infty} \phi^{1-\lambda}(x) f^{p}(x) d x
\end{aligned}
$$

In particular, when $p=q=2$, we have

$$
\begin{aligned}
\int_{0}^{\infty} \int_{0}^{\infty} \frac{f(x) g(y)}{(\phi(x)+\psi(y))^{\lambda}} d x d y \leq & \frac{B\left(\frac{\lambda}{2}, \frac{\lambda}{2}\right)}{\left(\inf \left\{\phi^{\prime}(x)\right\} \inf \left\{\psi^{\prime}(y)\right\}\right)^{\frac{1}{2}}} \\
& \times\left(\int_{0}^{\infty} \phi^{1-\lambda}(x) f^{2}(x) d x\right)^{\frac{1}{2}}\left(\int_{0}^{\infty} \psi^{1-\lambda}(y) g^{2}(y) d y\right)^{\frac{1}{2}} \\
\int_{0}^{\infty} \psi^{\lambda-1}(y)\left(\int_{0}^{\infty} \frac{f(x)}{(\phi(x)+\psi(y))^{\lambda}} d x\right)^{2} d y \leq & \frac{\left(B\left(\frac{\lambda}{2}, \frac{\lambda}{2}\right)\right)^{2}}{\left.\inf \left\{\phi^{\prime}(x)\right\}\right) \inf \left\{\psi^{\prime}(y)\right\}} \\
& \times \int_{0}^{\infty} \phi^{1-\lambda}(x) f^{2}(x) d x
\end{aligned}
$$

Proof. By Hölder's inequality and (10), we have

$$
\begin{aligned}
& \int_{0}^{\infty} \int_{0}^{\infty} \frac{f(x) g(y)}{(\phi(x)+\psi(y))^{\lambda}} d x d y \\
= & \int_{0}^{\infty} \int_{0}^{\infty} \frac{f(x)}{(\phi(x)+\psi(y))^{\frac{\lambda}{p}}}\left(\frac{\phi(x)}{\psi(y)}\right)^{\frac{2-\lambda}{p q}} \frac{g(y)}{(\phi(x)+\psi(y))^{\frac{\lambda}{q}}}\left(\frac{\psi(y)}{\phi(x)}\right)^{\frac{2-\lambda}{p q}} d x d y \\
\leq & \left(\int_{0}^{\infty} \int_{0}^{\infty} \frac{f^{p}(x)}{(\phi(x)+\psi(y))^{\lambda}}\left(\frac{\phi(x)}{\psi(y)}\right)^{\frac{2-\lambda}{p q}} d x d y\right)^{\frac{1}{p}} \\
& \times\left(\int_{0}^{\infty} \int_{0}^{\infty} \frac{g^{q}(y)}{(\phi(x)+\psi(y))^{\lambda}}\left(\frac{\psi(y)}{\phi(x)}\right)^{\frac{2-\lambda}{p}} d x d y\right)^{\frac{1}{q}} \\
= & \left(\int_{0}^{\infty} \omega_{1}(\phi, \psi, q, \lambda, x) f^{p}(x) d x\right)^{\frac{1}{p}}\left(\int_{0}^{\infty} \omega_{1}(\psi, \phi, q, \lambda, x) g^{q}(y) d y\right)^{\frac{1}{q}} \\
\leq & \frac{B\left(\frac{q+\lambda-2}{q}, \frac{p+\lambda-2}{p}\right)}{\left(\inf \left\{\phi^{\prime}(x)\right\}\right)^{\frac{1}{q}}\left(\inf \left\{\psi^{\prime}(y)\right\}\right)^{\frac{1}{p}}}\left(\int_{0}^{\infty} \phi^{1-\lambda}(x) f^{p}(x) d x\right)^{\frac{1}{p}}\left(\int_{0}^{\infty} \psi^{1-\lambda}(y) g^{q}(y) d y\right)^{\frac{1}{q}} .
\end{aligned}
$$

Hence (12) is valid. 
By Hölder's inequality and (10), we have

$$
\begin{aligned}
& \int_{0}^{\infty} \frac{f(x)}{(\phi(x)+\psi(y))^{\lambda}} d x \\
= & \int_{0}^{\infty} \frac{f(x)}{(\phi(x)+\psi(y))^{\frac{\lambda}{p}}}\left(\frac{\phi(x)}{\psi(y)}\right)^{\frac{2-\lambda}{p q}} \frac{1}{(\phi(x)+\psi(y))^{\frac{\lambda}{q}}}\left(\frac{\psi(y)}{\phi(x)}\right)^{\frac{2-\lambda}{p q}} d x \\
\leq & \left(\int_{0}^{\infty} \frac{f^{p}(x)}{(\phi(x)+\psi(y))^{\lambda}}\left(\frac{\phi(x)}{\psi(y)}\right)^{\frac{2-\lambda}{q}} d x\right)^{\frac{1}{p}}\left(\int_{0}^{\infty} \frac{1}{(\phi(x)+\psi(y))^{\lambda}}\left(\frac{\psi(y)}{\phi(x)}\right)^{\frac{2-\lambda}{p}} d x\right)^{\frac{1}{q}} \\
\leq & \left(\frac{B\left(\frac{q+\lambda-2}{q}, \frac{p+\lambda-2}{p}\right) \psi^{1-\lambda}(y)}{\inf \left\{\phi^{\prime}(x)\right\}}\right)^{\frac{1}{q}}\left(\int_{0}^{\infty} \frac{f^{p}(x)}{(\phi(x)+\psi(y))^{\lambda}}\left(\frac{\phi(x)}{\psi(y)}\right)^{\frac{2-\lambda}{q}} d x\right)^{\frac{1}{p}}
\end{aligned}
$$

Then

$$
\begin{aligned}
& \int_{0}^{\infty} \psi^{(\lambda-1)(p-1)}(y)\left(\int_{0}^{\infty} \frac{f(x)}{(\phi(x)+\psi(y))^{\lambda}} d x\right)^{p} d y \\
\leq & \left(\frac{B\left(\frac{q+\lambda-2}{q}, \frac{p+\lambda-2}{p}\right)}{\inf \left\{\phi^{\prime}(x)\right\}}\right)^{\frac{p}{q}} \int_{0}^{\infty} \int_{0}^{\infty} \frac{f^{p}(x)}{(\phi(x)+\psi(y))^{\lambda}}\left(\frac{\phi(x)}{\psi(y)}\right)^{\frac{2-\lambda}{q}} d x d y \\
\leq & \frac{\left(B\left(\frac{q+\lambda-2}{q}, \frac{p+\lambda-2}{p}\right)\right)^{p}}{\left(\inf \left\{\phi^{\prime}(x)\right\}\right)^{p-1} \inf \left\{\psi^{\prime}(y)\right\}} \int_{0}^{\infty} \phi^{1-\lambda}(x)(x) f^{p}(x) d x
\end{aligned}
$$

Hence (13) is valid. The theorem is proved.

Theorem 2. Let $p>1,1 / p+1 / q=1, f(x), g(x) \geq 0, \phi(x)$ and $\psi(x)$ are differentiable functions, and $\phi(0) \geq 0, \phi^{\prime}(x)>0, \psi(0) \geq 0, \psi^{\prime}(x)>0, \phi^{\prime}(x)$ and $\psi^{\prime}(x)$ has infimum, respectively, $\lambda>2-\min \{p, q\}$, such that $0<\int_{0}^{\infty} \phi^{1-\lambda}(t) f^{p}(t) d t<\infty$ and $0<\int_{0}^{\infty} \phi^{1-\lambda}(t) g^{q} d t<\infty$. Then

$$
\begin{aligned}
\int_{0}^{\infty} \int_{0}^{\infty} \frac{f(x) g(y)}{(\phi(x))^{\lambda}+(\psi(y))^{\lambda}} d x d y \leq & \frac{\left(B\left(\frac{q+\lambda-2}{q \lambda}, 1-\frac{q+\lambda-2}{q \lambda}\right)\right)^{\frac{1}{p}}\left(B\left(\frac{p+\lambda-2}{p \lambda}, 1-\frac{p+\lambda-2}{p \lambda}\right)\right)^{\frac{1}{q}}}{\lambda\left(\inf \left\{\phi^{\prime}(x)\right\}\right)^{\frac{1}{q}}\left(\inf \left\{\psi^{\prime}(y)\right\}\right)^{\frac{1}{p}}} \\
& \times\left(\int_{0}^{\infty} \phi^{1-\lambda}(x) f^{p}(x) d x\right)^{\frac{1}{p}}\left(\int_{0}^{\infty} \psi^{1-\lambda}(y) g^{q}(y) d y\right)^{\frac{1}{q}}(16) \\
& \int_{0}^{\infty} \psi^{(\lambda-1)(p-1)}(y)\left(\int_{0}^{\infty} \frac{f(x)}{(\phi(x))^{\lambda}+(\psi(y))^{\lambda}} d x\right)^{p} d y \\
\leq & \frac{\left(B\left(\frac{p+\lambda-2}{p \lambda}, 1-\frac{p+\lambda-2}{p \lambda}\right)\right)^{p-1} B\left(\frac{q+\lambda-2}{q \lambda}, 1-\frac{q+\lambda-2}{q \lambda}\right)}{\lambda^{p}\left(\inf \left\{\phi^{\prime}(x)\right\}\right)^{p-1} \inf \left\{\psi^{\prime}(y)\right\}} \int_{0}^{\infty} \phi^{1-\lambda}(x) f^{p}(x) d x .
\end{aligned}
$$


In particular, when $p=q=2$, we have

$$
\begin{aligned}
\int_{0}^{\infty} \int_{0}^{\infty} \frac{f(x) g(y)}{(\phi(x))^{\lambda}+(\psi(y))^{\lambda}} d x d y \leq & \frac{B\left(\frac{1}{2}, \frac{1}{2}\right)}{\lambda\left(\inf \left\{\phi^{\prime}(x)\right\} \inf \left\{\psi^{\prime}(y)\right\}\right)^{\frac{1}{2}}} \\
& \times\left(\int_{0}^{\infty} \phi^{1-\lambda}(x) f^{2}(x) d x\right)^{\frac{1}{2}}\left(\int_{0}^{\infty} \psi^{1-\lambda}(y) g^{2}(y) d y\right)^{\frac{1}{2}} \\
\int_{0}^{\infty} \psi^{\lambda-1}(y)\left(\int_{0}^{\infty} \frac{f(x)}{(\phi(x))^{\lambda}+(\psi(y))^{\lambda}} d x\right)^{2} d y \leq & \frac{\left(B\left(\frac{1}{2}, \frac{1}{2}\right)\right)^{2}}{\left.\lambda^{2} \inf \left\{\phi^{\prime}(x)\right\}\right) \inf \left\{\psi^{\prime}(y)\right\}} \\
& \times \int_{0}^{\infty} \phi^{1-\lambda}(x) f^{2}(x) d x
\end{aligned}
$$

Proof. By Hölder's inequality and (11), we have

$$
\begin{aligned}
& \int_{0}^{\infty} \int_{0}^{\infty} \frac{f(x) g(y)}{(\phi(x))^{\lambda}+(\psi(y))^{\lambda}} d x d y \\
= & \int_{0}^{\infty} \int_{0}^{\infty} \frac{f(x)}{\left((\phi(x))^{\lambda}+(\psi(y))^{\lambda}\right)^{\frac{1}{p}}}\left(\frac{\phi(x)}{\psi(y)}\right)^{\frac{2-\lambda}{p q}} \frac{g(y)}{\left((\phi(x))^{\lambda}+(\psi(y))^{\lambda}\right)^{\frac{1}{p}}}\left(\frac{\psi(y)}{\phi(x)}\right)^{\frac{2-\lambda}{p q}} d x d y \\
\leq & \left(\int_{0}^{\infty} \int_{0}^{\infty} \frac{f^{p}(x)}{(\phi(x))^{\lambda}+(\psi(y))^{\lambda}}\left(\frac{\phi(x)}{\psi(y)}\right)^{\frac{2-\lambda}{q}} d x d y\right)^{\frac{1}{p}} \\
& \times\left(\int_{0}^{\infty} \int_{0}^{\infty} \frac{g^{q}(y)}{(\phi(x))^{\lambda}+(\psi(y))^{\lambda}}\left(\frac{\psi(y)}{\phi(x)}\right)^{\frac{2-\lambda}{p}} d x d y\right)^{\frac{1}{q}} \\
= & \left(\int_{0}^{\infty} \omega_{2}(\phi, \psi, q, \lambda, x) f^{p}(x) d x\right)^{\frac{1}{p}}\left(\int_{0}^{\infty} \omega_{2}(\psi, \phi, q, \lambda, x) g^{q}(y) d y d y\right)^{\frac{1}{q}} \\
\leq & \frac{B\left(\frac{q+\lambda-2}{q \lambda}, \frac{p+\lambda-2}{p \lambda}\right)}{\lambda\left(\inf \left\{\phi^{\prime}(x)\right\}\right)^{\frac{1}{q}}\left(\inf \left\{\psi^{\prime}(y)\right\}\right)^{\frac{1}{p}}}\left(\int_{0}^{\infty} \phi^{1-\lambda}(x) f^{p}(x) d x\right)^{\frac{1}{p}}\left(\int_{0}^{\infty} \psi^{1-\lambda}(y) g^{q}(y) d y\right)^{\frac{1}{q}} .
\end{aligned}
$$

Hence (16) is valid.

By Hölder's inequality and (11), we have

$$
\begin{aligned}
& \int_{0}^{\infty} \frac{f(x)}{(\phi(x))^{\lambda}+(\psi(y))^{\lambda}} d x \\
= & \int_{0}^{\infty} \frac{f(x)}{\left((\phi(x))^{\lambda}+(\psi(y))^{\lambda}\right)^{\frac{1}{p}}}\left(\frac{\phi(x)}{\psi(y)}\right)^{\frac{2-\lambda}{p q}} \frac{1}{\left((\phi(x))^{\lambda}+(\psi(y))^{\lambda}\right)^{\frac{1}{q}}}\left(\frac{\psi(y)}{\phi(x)}\right)^{\frac{2-\lambda}{p q}} d x \\
\leq & \left(\int_{0}^{\infty} \frac{f^{p}(x)}{(\phi(x))^{\lambda}+(\psi(y))^{\lambda}}\left(\frac{\phi(x)}{\psi(y)}\right)^{\frac{2-\lambda}{q}} d x\right)^{\frac{1}{p}}\left(\int_{0}^{\infty} \frac{1}{(\phi(x))^{\lambda}+(\psi(y))^{\lambda}}\left(\frac{\psi(y)}{\phi(x)}\right)^{\frac{2-\lambda}{p}} d x\right)^{\frac{1}{q}}
\end{aligned}
$$




$$
\leq\left(\frac{B\left(\frac{p+\lambda-2}{p \lambda}, 1-\frac{p+\lambda-2}{p \lambda}\right) \psi^{1-\lambda}(y)}{\lambda \inf \left\{\phi^{\prime}(x)\right\}}\right)^{\frac{1}{q}}\left(\int_{0}^{\infty} \frac{f^{p}(x)}{(\phi(x))^{\lambda}+(\psi(y))^{\lambda}}\left(\frac{\phi(x)}{\psi(y)}\right)^{\frac{2-\lambda}{q}} d x\right)^{\frac{1}{p}} .
$$

Then

$$
\begin{aligned}
& \int_{0}^{\infty} \psi^{(\lambda-1)(p-1)}(y)\left(\int_{0}^{\infty} \frac{f(x)}{(\phi(x))^{\lambda}+(\psi(y))^{\lambda}} d x\right)^{p} d y \\
\leq & \left(\frac{B\left(\frac{p+\lambda-2}{p \lambda}, 1-\frac{p+\lambda-2}{p \lambda}\right)}{\lambda \inf \left\{\phi^{\prime}(x)\right\}}\right)^{\frac{p}{q}} \int_{0}^{\infty} \int_{0}^{\infty} \frac{f^{p}(x)}{(\phi(x))^{\lambda}+(\psi(y))^{\lambda}}\left(\frac{\phi(x)}{\psi(y)}\right)^{\frac{2-\lambda}{q}} d x d y \\
\leq & \frac{\left(B\left(\frac{p+\lambda-2}{p \lambda}, 1-\frac{p+\lambda-2}{p \lambda}\right)\right)^{p-1} B\left(\frac{q+\lambda-2}{q \lambda}, 1-\frac{q+\lambda-2}{q \lambda}\right)}{\lambda^{p}\left(\inf \left\{\phi^{\prime}(x)\right\}\right) \inf \left\{\psi^{\prime}(y)\right\}} \int_{0}^{\infty} \phi^{1-\lambda}(x) f^{p}(x) d x .
\end{aligned}
$$

Hence (17) is valid. The theorem is proved.

Theorem 3. Let $p>1,1 / p+1 / q=1,\left\{a_{m}\right\}$ and $\left\{b_{n}\right\}$ are two arbitary sequences of nonnegative real numbers. $\phi(x)$ and $\psi(y)$ be differentiable functions, and $\phi(0) \geq 0$, $\phi^{\prime}(x)>0, \psi(0) \geq 0, \psi^{\prime}(x)>0, \phi^{\prime}(x)$ and $\psi^{\prime}(x)$ has infimum, respectively, $2 \geq \lambda>$ $2-\min \{p, q\}$, such that $0<\sum_{m=1}^{\infty}(\phi(m))^{1-\lambda} a_{m}<\infty$ and $0<\sum_{n=1}^{\infty}(\psi(n))^{1-\lambda} b_{n}<\infty$. Then

$$
\begin{aligned}
\sum_{m=1}^{\infty} \sum_{n=1}^{\infty} \frac{a_{m} b_{n}}{(\phi(m)+\psi(n))^{\lambda}} \leq & \frac{B\left(\frac{q+\lambda-2}{q}, \frac{p+\lambda-2}{p}\right)}{\left(\inf \left\{\phi^{\prime}(x)\right\}\right)^{\frac{1}{q}}\left(\inf \left\{\psi^{\prime}(y)\right\}\right)^{\frac{1}{p}}} \\
& \times\left(\sum_{m=1}^{\infty} \phi^{1-\lambda}(m) a_{m}^{p}\right)^{\frac{1}{p}}\left(\sum_{n=1}^{\infty} \psi^{1-\lambda}(n) b_{n}^{q}\right)^{\frac{1}{q}} \\
\sum_{n=1}^{\infty} \psi^{(\lambda-1)(p-1)}(n)\left(\sum_{m=1}^{\infty} \frac{a_{m}}{(\phi(m)+\psi(n))^{\lambda}}\right)^{p}< & \frac{\left(B\left(\frac{q+\lambda-2}{q}, \frac{p+\lambda-2}{p}\right)\right)^{p}}{\left(\inf \left\{\phi^{\prime}(x)\right\}\right)^{p-1} \inf \left\{\psi^{\prime}(y)\right\}} \\
& \times \sum_{m=1}^{\infty} \phi^{1-\lambda}(m) a_{m}^{p} .
\end{aligned}
$$

Proof. By Hölder's inequality, we have

$$
\begin{aligned}
& \sum_{m=1}^{\infty} \sum_{n=1}^{\infty} \frac{a_{m} b_{n}}{(\phi(m)+\psi(n))^{\lambda}} \\
= & \sum_{m=1}^{\infty} \sum_{n=1}^{\infty} \frac{a_{m}}{(\phi(m)+\psi(n))^{\frac{\lambda}{p}}}\left(\frac{\phi(m)}{\psi(n)}\right)^{\frac{2-\lambda}{p q}} \frac{b_{n}}{(\phi(m)+\psi(n))^{\frac{\lambda}{q}}}\left(\frac{\psi(n)}{\phi(m)}\right)^{\frac{2-\lambda}{p q}} \\
\leq & \left(\sum_{m=1}^{\infty} \sum_{n=1}^{\infty} \frac{a_{m}^{p}}{(\phi(m)+\psi(n))^{\lambda}}\left(\frac{\phi(m)}{\psi(n)}\right)^{\frac{2-\lambda}{q}}\right)^{\frac{1}{p}}
\end{aligned}
$$




$$
\begin{aligned}
& \times\left(\sum_{m=1}^{\infty} \sum_{n=1}^{\infty} \frac{b_{n}^{q}}{(\phi(m)+\psi(n))^{\lambda}}\left(\frac{\psi(n)}{\phi(m)}\right)^{\frac{2-\lambda}{p}}\right)^{\frac{1}{q}} \\
= & \left(\sum_{m=1}^{\infty} \omega_{3}(\phi, \psi, q, \lambda, m) a_{m}^{p}\right)^{\frac{1}{p}}\left(\sum_{n=1}^{\infty} \omega_{3}(\psi, \phi,, p, \lambda, n) b_{n}^{q}\right)^{\frac{1}{q}}
\end{aligned}
$$

where $\omega_{3}(\phi, \psi, q, \lambda, m)=\sum_{n=1}^{\infty} \frac{1}{(\phi(m)+\psi(n))^{\lambda}}\left(\frac{\phi(m)}{\psi(n)}\right)^{\frac{2-\lambda}{q}}$.

By (10), we have

$$
\begin{aligned}
\omega_{3}(\phi, \psi, q, \lambda, m) & <\int_{0}^{\infty} \frac{1}{(\phi(m)+\psi(y))^{\lambda}}\left(\frac{\phi(m)}{\psi(y)}\right)^{\frac{2-\lambda}{q}} d y \\
& \leq \frac{\phi^{1-\lambda}(m)}{\inf \left\{\psi^{\prime}(y)\right\}} B\left(\frac{q+\lambda-2}{q}, \frac{p+\lambda-2}{p}\right) .
\end{aligned}
$$

Hence (20) is valid.

By Hölder's inequality and (22), we have

$$
\begin{aligned}
& \sum_{m=1}^{\infty} \frac{a_{m}}{(\phi(m)+\psi(n))^{\lambda}} \\
= & \sum_{m=1}^{\infty} \frac{a_{m}}{(\phi(m)+\psi(n))^{\frac{\lambda}{p}}}\left(\frac{\phi(m)}{\psi(n)}\right)^{\frac{2-\lambda}{p q}} \frac{1}{(\phi(m)+\psi(n))^{\frac{\lambda}{q}}}\left(\frac{\psi(n)}{\phi(m)}\right)^{\frac{2-\lambda}{p q}} \\
\leq & \left(\sum_{m=1}^{\infty} \frac{a_{m}^{p}}{(\phi(m)+\psi(n))^{\lambda}}\left(\frac{\phi(m)}{\psi(n)}\right)^{\frac{2-\lambda}{q}}\right)^{\frac{1}{p}}\left(\sum_{m=1}^{\infty} \frac{1}{(\phi(m)+\psi(n))^{\lambda}}\left(\frac{\psi(n)}{\phi(m)}\right)^{\frac{2-\lambda}{p}}\right)^{\frac{1}{q}} \\
< & \left(\frac{B\left(\frac{q+\lambda-2}{q}, \frac{p+\lambda-2}{p}\right) \psi^{1-\lambda}(n)}{\inf \left\{\phi^{\prime}(x)\right\}}\right)^{\frac{1}{q}}\left(\sum_{m=1}^{\infty} \frac{a_{m}^{p}}{(\phi(m)+\psi(n))^{\lambda}}\left(\frac{\phi(m)}{\psi(n)}\right)^{\frac{2-\lambda}{q}}\right)^{\frac{1}{p}} .
\end{aligned}
$$

Then

$$
\begin{aligned}
& \sum_{n=1}^{\infty} \psi^{(\lambda-1)(p-1)}(n)\left(\sum_{m=1}^{\infty} \frac{a_{m}}{(\phi(m)+\psi(n))^{\lambda}}\right)^{p} \\
< & \left(\frac{B\left(\frac{q+\lambda-2}{q}, \frac{p+\lambda-2}{p}\right)}{\inf \left\{\phi^{\prime}(x)\right\}}\right)^{\frac{p}{q}} \sum_{n=1}^{\infty} \sum_{m=1}^{\infty} \frac{a_{m}^{p}}{(\phi(m)+\psi(n))^{\lambda}}\left(\frac{\phi(m)}{\psi(n)}\right)^{\frac{2-\lambda}{q}} \\
< & \frac{\left(B\left(\frac{q+\lambda-2}{q}, \frac{p+\lambda-2}{p}\right)\right)^{p}}{\left(\inf \left\{\phi^{\prime}(x)\right\}\right)^{p-1} \inf \left\{\psi^{\prime}(y)\right\}} \sum_{m=1}^{\infty} \phi^{1-\lambda}(m) a_{m}^{p} .
\end{aligned}
$$

Hence (21) is valid. The theorem is proved.

In a similar way to the proof of Theorem 3, the following Theorem 4 can be showed. 
Theorem 4. Let $p>1,1 / p+1 / q=1,\left\{a_{m}\right\}$ and $\left\{b_{n}\right\}$ be two arbitary sequences of nonnegative real numbers. $\phi(x)$ and $\psi(y)$ be differentiable functions, and $\phi(0) \geq 0$, $\phi^{\prime}(x)>0, \psi(0) \geq 0, \psi^{\prime}(x)>0, \phi^{\prime}(x)$ and $\psi^{\prime}(x)$ has infimum, respectively, $2 \geq \lambda>$ $2-\min \{p, q\}$, such that $0<\sum_{m=1}^{\infty}(\phi(m))^{1-\lambda} a_{m}<\infty$ and $0<\sum_{n=1}^{\infty}(\psi(n))^{1-\lambda} b_{n}<\infty$. Then

$$
\begin{aligned}
\sum_{m=1}^{\infty} \sum_{n=1}^{\infty} \frac{a_{m} b_{n}}{(\phi(m))^{\lambda}+(\psi(n))^{\lambda}}< & \frac{\left(B\left(\frac{q+\lambda-2}{q \lambda}, 1-\frac{q+\lambda-2}{q \lambda}\right)\right)^{\frac{1}{p}}\left(B\left(\frac{p+\lambda-2}{p \lambda}, 1-\frac{p+\lambda-2}{p \lambda}\right)\right)^{\frac{1}{q}}}{\lambda\left(\inf \left\{\phi^{\prime}(x)\right\}\right)^{\frac{1}{q}}\left(\inf \left\{\psi^{\prime}(y)\right\}\right)^{\frac{1}{p}}} \\
& \times\left(\sum_{m=1}^{\infty} \phi^{1-\lambda}(m) a_{m}^{p}\right)^{\frac{1}{p}}\left(\sum_{n=1}^{\infty} \psi^{1-\lambda}(n) b_{n}^{q}\right)^{\frac{1}{q}} \\
& \sum_{n=1}^{\infty} \psi^{(\lambda-1)(p-1)}(n)\left(\sum_{m=1}^{\infty} \frac{a_{m}}{(\phi(m))^{\lambda}+(\psi(n))^{\lambda}}\right)^{p} \\
< & \frac{\left(B\left(\frac{p+\lambda-2}{p \lambda}, 1-\frac{p+\lambda-2}{p \lambda}\right)\right)^{p-1} B\left(\frac{q+\lambda-2}{q \lambda}, 1-\frac{q+\lambda-2}{q \lambda}\right)}{\lambda^{p}\left(\inf \left\{\phi^{\prime}(x)\right\}\right)^{p-1} \inf \left\{\psi^{\prime}(y)\right\}} \sum_{m=1}^{\infty} \phi^{1-\lambda}(m) a_{m}^{p}
\end{aligned}
$$

Remark 1. For $\phi(x)=A x, \psi(y)=B y, A>0, B>0$, inequalities (14) and (15) change to (6) and (7), respectively, hence inequalities (14) and (15) are generalizations of (6) and (7), respectively.

Remark 2. For $\phi(x)=A x, \psi(y)=B y, A>0, B>0, p=q=2$, inequalities (20) and (21) change to (8) and (9), respectively, hence inequalities (20) and (21) are generalizations of (8) and (9), respectively.

Remark 3. For $\phi(x)=x, \psi(y)=y, p=q=2$, inequalities (12), (14), (16) and (18) change to (5), hence inequalities (12), (14), (16) and (18) are generalizations of (5).

Remark 4. For $\phi(x)=x, \psi(y)=y, p=q=2, \lambda=1$, inequalities (23) and (24) change to (3) and (4), respectively, hence inequalities (23) and (24) are generalizations of (3) and (4), respectively.

\section{References}

[1] G. H. Hardy, J. E. Littlewood and G. Polya, Inequalities, Cambridge Univ. Press, London, 1952.

[2] $\mathrm{Hu} \mathrm{Ke}$, On of Hilbert's inequality, Chinese Ann. of Math. 13B(1992), 35-39.

[3] M. Gao, Tan Li and L. Debnath, Some improvements on Hilbert's integral inequality, J. Math. Anal. Appl. 229(1999), 682-289.

[4] J. Kuang, On new extensions of Hilbert's integral inequality, J. Math. Anal. Appl. 235 (1999), 608-614. 
[5] M. Gao, On Hilbert's inequality and its applications, J. Math. Anal. Appl. 212(1997), 316-323.

[6] B. Yang, A generalized Hilbert's integral inequality with the best const, Ann. of Math. (Chinese) 21A(2000), 401-408.

[7] B. Yang, On new generalizations of Hilbert's inequality, J. Math. Anal. Appl. 248(2000), 29-40.

[8] Yang Bicheng, On generalization of Hardy-Hilbert's integral inequality, Acta Math Sinica (China) 41(1998), 839-844, (in Chinese).

[9] B. Yang, On Hilbert's integral inequality, J. Math. Anal. Appl. 220(1998), 778-785.

[10] J. Kuang, Applied Inequalities, 2nd ed., Hunan Jiaoyu Chubanshe, Changsha, 1993 (Chinese). MR 95j:26001.

Department of Basic Science of Technology College, Xuzhou Normal University, Xuzhou, Jiangsu, 221011, P.R.C.

E-mail: lvzx1@163.net

School of Science, Nanjing University of Science \& Technology, Nanjing 210094, P.R.C. 